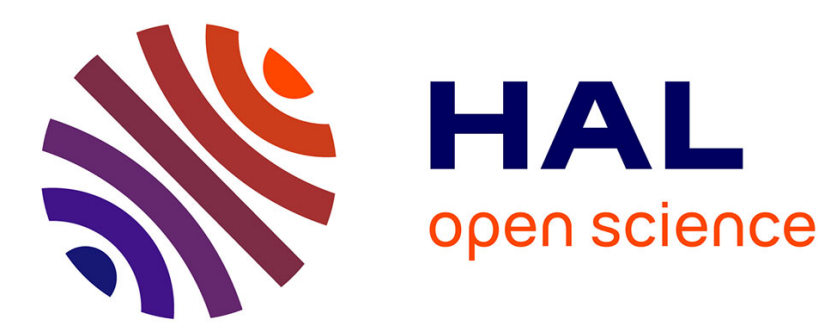

\title{
A Claims Persistence Process and Insurance
}

Pierre P. Vallois, Charles S. Tapiero

\section{- To cite this version:}

Pierre P. Vallois, Charles S. Tapiero. A Claims Persistence Process and Insurance. Insurance: Mathematics and Economics, 2009, 44 (3), pp.367-373. 10.1016/j.insmatheco.2008.11.009 . hal-00591746

\section{HAL Id: hal-00591746 \\ https://hal.science/hal-00591746}

Submitted on 10 May 2011

HAL is a multi-disciplinary open access archive for the deposit and dissemination of scientific research documents, whether they are published or not. The documents may come from teaching and research institutions in France or abroad, or from public or private research centers.
L'archive ouverte pluridisciplinaire HAL, est destinée au dépôt et à la diffusion de documents scientifiques de niveau recherche, publiés ou non, émanant des établissements d'enseignement et de recherche français ou étrangers, des laboratoires publics ou privés. 


\section{Accepted Manuscript}

A claims persistence process and insurance

Pierre Vallois, Charles S. Tapiero

PII:

S0167-6687(08)00164-9

DOI: $\quad$ 10.1016/j.insmatheco.2008.11.009

Reference: INSUMA 1346

To appear in: Insurance: Mathematics and Economics

Received date: May 2008

Revised date: September 2008

Accepted date: 13 November 2008

Please cite this article as: Vallois, P., Tapiero, C.S., A claims persistence process and insurance. Insurance: Mathematics and Economics (2008), doi:10.1016/j.insmatheco.2008.11.009

This is a PDF file of an unedited manuscript that has been accepted for publication. As a service to our customers we are providing this early version of the manuscript. The manuscript will undergo copyediting, typesetting, and review of the resulting proof before it is published in its final form. Please note that during the production process errors may be discovered which could affect the content, and all legal disclaimers that apply to the journal pertain. 
Revised

September 27, 2008

\title{
A Claims Persistence Process and Insurance
}

\author{
Pierre Vallois
}

Département de Mathématiques, Université de Nancy I

B.P. 239 - 54506 Vandoeuvre les Nancy Cedex - France

Charles S. Tapiero,

Department of Finance and Risk Engineering

New York University Polytechnic Institute, New York

Keywords : Random walk, Persistence, Insurance Claims, Value at Risk 


\begin{abstract}
The purpose of this paper is to introduce and construct a state dependent counting and persistent random walk. Persistence is imbedded in a Markov chain for predicting insured claims based on their current and past period claim. We calculate for such a process the probability generating function of the number of claims over time and as a result are able to calculate their moments. Further, given the claims severity probability distribution, we provide both the claim's process generating function as well as the mean and the claim variance that an insurance firm confronts over a given period of time and in such circumstances. A number of results and applictions are then outlined (such as a Compount Claim Persistence Process).
\end{abstract}




\section{Introduction}

Insured claims may be time dependent. For example, an insured who has claimed one year might be more careful the following year and thereby alter the probability that a claim occurs. By the same token, an insured whose health has been impaired one year may be more prone to claim subsequently. Such persistence recurs in many instances motivated and implied in insured attitudes and expectations as well as recurring due to events inherent to the claim process (see for example Denuit, et al., 2006). Questions such as, does car failure induce additional and subsequent car failures? Are terrorist attacks correlated (Telesca and Lovallo, 2006), one attack defining the propensity for a subsequent attack or a respite ? Does the severity of hurricanes one year imply the severity of the following year? etc., are obvously questions of importance for insurers. Similarly, does a patient relapses following a treatment or not? Do stock prices have an inertia, tending to increase (or decrease) following increases or decreases with the same probabilities? Are fear regarding financial markets persistent? (The Financial Times, February 7, 2008, p.28). Most actuarial counting approaches assume a Poisson distribution, implying that events are independent. In other words, prior events do not alter the basic probabilities laws that determine the occurrence of subsequent events. To circumvent this lacuna, the credibility theory approach in actuarial science, evaluates the objectivity and the subjectivity of a sourcethe insured that may potentially claim, and devises a statistical "learning" meachanism that allows the updating of the underlying claim probability. Using Bayesian statistics for example, credibility theory divides insured into classes that have various propensities to claim, which are updated using subjective prior estimates of risk classes and an accrued experience-the claim history of insured which is observed. The goal of credibility theory is then to set up an experience rating system to determine next year's premium, accounting for the individual and the collective group experience. Unlike credibility theory, this paper presumes that there may be an inherent persistence in an underlying process that will dictate the probability laws with which subsequent events occur. The probability of a subsequent claim for example, will then be determined by the past memory (in our case, the single past event of a claim or no claim) rather than be determined by a statistical estimator based on the accrued evidence of past claims. Explicitly, while credibility theory seeks to integrate "experience" in estimating the propensity to claim, a persistence to claim is an inherent property of the underlying 
claiming (stochastic process) that determines, conditionally on the "claim memory", the actual probabilities with which an insured will claim or not. For example, if an insured claiming (or not) one year will be more careful (or less careful) the following year, then the underlying claiming process will account for such a behavior in defining the effects of the "process memory" on the claiming stochastic process. The credibility approach however, will use the fact that a claim has been made to revise the probability that he will claim again in the following year with a credibly (larger) probability. In this sense these approaches differ fundamentally.

The purpose of this paper is to consider such a counting persistent process based on the Markov memory of the immediate and past event (and not only its state) and claculate the generating function of such a process (for example, see Patlak [1953], Weiss and Rubin [1983], Balinth [1986], Claes and Van den Brock [1987], Weiss [1994, 2002], Pottier [1996], Vallois and Tapiero [2007] for prior research on such processes). The resulting persistent counting process will be shown on the one hand to depart from the Poisson counting process and recognize the effects of the past memory on the claim process. Given such a process, we assess the effects of persistent claims on an insurance contract and use such observation to better assess the risk premium needed to compensate its risk exposure. The result obtain in this paper extend the results obtained in Vallois and Tapiero [2007]. Explicitly, we extend our previous result by providing the probability generating function of the persistent claim process, its moments as well as an explicit expresison for the probabilities of such a process. In particular, the process kurtosis due to persistence is expressed explicitly. Finally, a general expression for Persistent Compound processes is provided and an explicit recursive equations for its probability moments is given explicitly, generalizing thereby the often used Compount Poisson process. Numerical analyses are then used to highlight the effects of persistence counting compared to traditional counting and claiming processes.

\section{The Markov Memory Based Persistent Counting Process}

Assume that a representative insured can in any one year claim or not, denoting these events by $(0,1)$. The event « 0 » states that no claim is made within the year while a « 1 » states that the insured has filed a claim during the year. Claim records indicate that when a claim is made in a given year, then the following year, the probability of no claim is $\beta>0$. When a claim is not made 
in a given year, then the probability of a claim being made in the following year is $\alpha$. This defines a simple two-states Markov chain, given by:

(1) $\mathbf{P}=\left[\begin{array}{cc}1-\alpha & \alpha \\ \beta & 1-\beta\end{array}\right], 0<\alpha, \beta<1$

If we denote by $y_{t}$ the values the random event can assume at time then:

(2) $\left\{\begin{array}{l}P\left(y_{t}=1 \mid y_{t-1}=0\right)=\alpha, P\left(y_{t}=0 \mid y_{t-1}=0\right)=1-\alpha \\ P\left(y_{t}=1 \mid y_{t-1}=1\right)=1-\beta, P\left(y_{t}=0 \mid y_{t-1}=1\right)=\beta\end{array}\right.$

Or

(3) $\left[\begin{array}{ll}P\left(y_{t}=0\right) & P\left(y_{t}=1\right)\end{array}\right]=\left[\begin{array}{ll}P\left(y_{t-1}=0\right) & P\left(y_{t-1}=1\right)\end{array}\right]\left[\begin{array}{cc}1-\alpha & \alpha \\ \beta & 1-\beta\end{array}\right]$

Over a period of time t, the total number of events (claims made, etc.) is therefore given by:

(4) $x_{t}=\sum_{j=0}^{t} y_{j}$

The number of claims are conditional on the initial event (the current memory of the event), denoted by: $y_{0}=0$ or $y_{0}=1$. In this memory-based persistent counting process, we calculate first the probability generating function (PGF), summarized in Proposition 1 and subsequently use this PGF to obtain some of the characteristics of an underlying claim process.

\section{Proposition 1}

Let $\left\{x_{t}, t \geq 0\right\}$ be a counting random variable of the number of claim events in a time interval $(0, t)$. And let $(\alpha, 1-\beta)$ be the probabilities that an event occurs at time $t$, conditional on its current (or not) occurrence in the previous period $t-1$. Define $\rho=1-\alpha-\beta, \rho \in]-1,1[$, as a "persistence index. Let $G(\lambda, t)=E\left\{\lambda^{x_{t}}\right\}$ be the probability generating function for the persistent counting process, for counting the number of claims, given by equation (4), in a time interval $(0, t)$ and let $P\left(x_{0}=0\right)$ be the probability that initially no claim is made while $P\left(x_{0}=1\right)$ denotes the probability that a claim was made initially and set for notational convenience. Then: 
(5) $G(\lambda, t)=\frac{G(\lambda, 1)}{\sqrt{\delta}} \Psi_{t}-\lambda \rho \frac{G(\lambda, 0)}{\sqrt{\delta}} \Psi_{t-1}, t \geq 1$

with

(6) $G(\lambda, 0)=P\left(x_{0}=0\right)+\lambda P\left(x_{0}=1\right)$

$G(\lambda, 1)=(1-\alpha+\lambda \alpha) P\left(x_{0}=0\right)+\lambda(1+(\lambda-1)(\alpha+\rho)) P\left(x_{0}=1\right)$

where

$$
\begin{aligned}
& a=1-\alpha+\lambda \alpha, \\
& \delta=(a+\lambda \rho)^{2}-4 \lambda \rho \\
& \Psi_{t}=\mu_{0}^{t}-\mu_{1}^{t} \\
& \mu_{0}=\frac{1}{2}(a+\lambda \rho+\sqrt{\delta}), \\
& \mu_{1}=\frac{1}{2}(a+\lambda \rho-\sqrt{\delta})
\end{aligned}
$$

Proof: See Appendix 1

Such a generating function provides the means to calculate higher order moments of the underlying persistent counting claim process as well as the probabilities of the number of claims. Further, it clearly points out to the effects of the persistence index on the counting process. When $\rho=0$ then $\beta=1-\alpha$ and whatever the previous outcome (whether a claim or no claim), the subsequent probability to claim is $\alpha$ while that of no claim is $1-\alpha$. When the persistence index is positive, $\rho>0$ then $\beta=1-\alpha-\rho$. That is, if in a given year a claim is made, then the probability that in a subsequent year a claim is made has a smaller probability. And vice versa, when the persistence index is negative $\rho<0$ and the underlying stochastic process would point out to a "contagious" claim process (for example, with Hurricanes of a high category following Hurricanes of High category). Inversely, for a positive persistence index $\rho>0$ it will indicate that the underlying claim process has a built-in "incentive effect", reducing a claim probability in a given year following a claim made in the previous one. 
Our results in proposition 1 allow an explicit calculation of the moments of the persistent counting process, defined recursively as shown below. Note that:

$\frac{\partial G}{\partial \lambda}(\lambda, t)=E\left(x_{t} \lambda^{x_{t}-1}\right), \quad \frac{\partial^{2} G}{\partial \lambda^{2}}(\lambda, t)=E\left(x_{t}\left(x_{t}-1\right) \lambda^{x_{t}-2}\right), \quad \frac{\partial^{3} G}{\partial \lambda^{3}}(\lambda, t)=E\left(x_{t}\left(x_{t}-1\right)\left(x_{t}-2\right) \lambda^{x_{t}-3}\right)$ and so on for higher order terms. Using these terms and setting $\lambda=1$, we obtain the necessary equations which allow the calculation of the mean, the variance, the kurtosis and other moments of the persisting counting process distribution. It is also useful to derive a number of special and well known cases to confirm the validity of our results. First note that when $\lambda=1$ then $G(1, t)=1$ as expected. Further, when there is no persistence (i.e $\rho=0$ ), we have $G(\lambda, t)=\frac{G(\lambda, 1)}{a} \Psi_{t}$, $\mu_{0}=a, \mu_{1}=0$ and $\Psi_{t}=a^{t}$ and therefore:

(7) $G(\lambda, t)=\left\{P\left(x_{0}=0\right)+\lambda P\left(x_{0}=1\right)\right\} a^{t}$

In particular, if initially, $P\left(x_{0}=0\right)=1$, then $G(\lambda, t)=a^{t}$ which corresponds as expected to the Probability Generating Function of a binomial distribution. However, if $P\left(x_{0}=1\right)=1$, then $G(\lambda, t)=\lambda a^{t}$.

A convenient recursive expression for the generating function can be found by noting that $\Psi_{t}=\mu_{0}{ }^{t}-\mu_{1}^{t}$ where $\mu_{0}$ and $\mu_{1}$ solve $\mu^{2}-\mu(\lambda(\rho+\alpha)+1-\alpha)+\lambda \rho=0$, (proved in Appendix 1) and verify the second order equation:

(8) $\Psi_{t+2}-(a+\lambda \rho) \Psi_{t+1}+\lambda \rho \Psi_{t}=0$

As a result, (5) implies that the probability generating function $G(\lambda, t)$ satisfies as well the second order recursive equation given by:

(9) $G(\lambda, t+2)=(1-\alpha+\lambda(\alpha+\rho)) G(\lambda, t+1)-\lambda \rho G(\lambda, t)$

Deriving (9) with respect to $\lambda$ with $\lambda=1$ we obtain a recursive expression for the moments of the counting process. Concentrating our attention on the first moments only, derivatives of (9) yields the following recursive equation:

$$
\begin{aligned}
G(\lambda, t+2)=(1-\alpha+\lambda(\alpha+\rho)) G(\lambda, t+1)-\lambda \rho G(\lambda, t) \\
\text { (10) } \frac{\partial^{k} G(\lambda, t+2)}{\partial \lambda^{k}}=(1-\alpha+\lambda(\alpha+\rho)) \frac{\partial^{k} G(\lambda, t+1)}{\partial \lambda^{k}}-\lambda \rho \frac{\partial^{k} G(\lambda, t)}{\partial \lambda^{k}} \\
+k(\alpha+\rho) \frac{\partial^{k-1} G(\lambda, t+1)}{\partial \lambda^{k-1}}-k \rho \frac{\partial^{k-1} G(\lambda, t)}{\partial \lambda^{k-1}}, k=1,2,3, \ldots
\end{aligned}
$$


With initial conditions:

$$
\begin{aligned}
G(\lambda, 0)=P\left(x_{0}=0\right)+\lambda P\left(x_{0}=1\right) \\
G(\lambda, 1)=(1-\alpha+\lambda \alpha) P\left(x_{0}=0\right)+\lambda(1+(\lambda-1)(\alpha+\rho)) P\left(x_{0}=1\right) \\
\text { (11) } \frac{\partial G(\lambda, 0)}{\partial \lambda}=P\left(x_{0}=1\right), \quad \frac{\partial G(\lambda, 1)}{\partial \lambda}=\alpha P\left(x_{0}=0\right)+(1+(2 \lambda-1)(\alpha+\rho)) P\left(x_{0}=1\right) \\
\frac{\partial^{2} G(\lambda, 0)}{\partial \lambda^{2}}=0, \quad \frac{\partial^{2} G(\lambda, 1)}{\partial \lambda^{2}}=2(\alpha+\rho) P\left(x_{0}=1\right) \\
\frac{\partial^{j} G(\lambda, 0)}{\partial \lambda^{j}}=0, \quad \frac{\partial^{j} G(\lambda, 1)}{\partial \lambda^{j}}=0, \text { for } j \geq 3
\end{aligned}
$$

These initial conditions are specified using equation (6) in Proposition1. At $\lambda=1$, we can write these expressions in the following manner (together with equation (9)) which simplifies their numerical solution:

$$
G(1, t+2)=(1+\rho) G(1, t+1)-\rho G(1, t)
$$

(12) $\frac{\partial^{k} G(1, t+2)}{\partial \lambda^{k}}=(1+\rho) \frac{\partial^{k} G(1, t+1)}{\partial \lambda^{k}}-\rho \frac{\partial^{k} G(1, t)}{\partial \lambda^{k}}+k(\alpha+\rho) \frac{\partial^{k-1} G(1, t+1)}{\partial \lambda^{k-1}}-k \rho \frac{\partial^{k-1} G(1, t)}{\partial \lambda^{k-1}}$,

While the initial conditions stated above in equation (11) and leading to:

$$
\begin{aligned}
& G(1,0)=1, \quad G(1,1)=1 \\
& \frac{\partial G(1,0)}{\partial \lambda}=P\left(x_{0}=1\right), \quad \frac{\partial G(1,1)}{\partial \lambda}=\alpha+(1+\rho) P\left(x_{0}=1\right) \\
& \frac{\partial^{2} G(1,0)}{\partial \lambda^{2}}=0, \quad \frac{\partial^{2} G(1,1)}{\partial \lambda^{2}}=2(\alpha+\rho) P\left(x_{0}=1\right) \\
& \frac{\partial^{j} G(1,0)}{\partial \lambda^{j}}=0, \quad \frac{\partial^{j} G(1,1)}{\partial \lambda^{j}}=0, \text { for } j \geq 3 .
\end{aligned}
$$

Similarly, we can calculate the probabilities of persistent counting process by setting $\lambda=0$ in the derivatives of the generating functions. In this case, the probabilities are given by:

$$
\begin{aligned}
& p_{i}(t)=\frac{1}{i !} \frac{\partial^{i} G(\lambda, t)}{\partial \lambda^{i}}, i=0,1,2,3, \ldots, t \text { with: } \\
& G(0, t+2)=(1-\alpha) G(0, t+1) \\
& \begin{aligned}
(14) \frac{\partial^{k} G(0, t+2)}{\partial \lambda^{k}} & =(1-\alpha) \frac{\partial^{k} G(0, t+1)}{\partial \lambda^{k}} \\
& +k(\alpha+\rho) \frac{\partial^{k-1} G(0, t+1)}{\partial \lambda^{k-1}}-k \rho \frac{\partial^{k-1} G(0, t)}{\partial \lambda^{k-1}},
\end{aligned}
\end{aligned}
$$

With the initial conditions (specified by equation (6)): 
(15)

$$
\begin{aligned}
& G(0,0)=P\left(x_{0}=0\right), G(0,1)=(1-\alpha) P\left(x_{0}=0\right) \\
& \frac{\partial G(0,0)}{\partial \lambda}=P\left(x_{0}=1\right), \quad \frac{\partial G(0,1)}{\partial \lambda}=\alpha P\left(x_{0}=0\right)+(1-\alpha-\rho) P\left(x_{0}=1\right) \\
& \frac{\partial^{2} G(0,0)}{\partial \lambda^{2}}=0, \quad \frac{\partial^{2} G(0,1)}{\partial \lambda^{2}}=2(\alpha+\rho) P\left(x_{0}=1\right) \\
& \frac{\partial^{j} G(\lambda, 0)}{\partial \lambda^{j}}=0, \quad \frac{\partial^{j} G(\lambda, 1)}{\partial \lambda^{j}}=0, \text { for } j \geq 3 .
\end{aligned}
$$

These equations define a numerical approach to calculating both the moments and the probabilities of a persistent process. A more direct approach will be outlined subsequently however.

Explicit results for the first two moments are provided below with proofs found directly from equations (12) and (13).

\section{Proposition 2}

Let $x_{0}=0$, then:

$$
\text { (16) } \begin{aligned}
E(x(t)) & =(1+\rho) E(x(t-1))-\rho E(x(t-2))+\alpha, t \geq 2 \\
E\left(x(t)^{2}\right) & =(1+\rho) E\left(x(t-1)^{2}\right)-\rho E\left(x(t-2)^{2}\right)+ \\
& \text { (17) } \quad \frac{\alpha}{1-\rho}\left[2 \alpha t+\rho+1-2\left(\frac{\alpha+(1-\alpha) \rho^{t}-\rho^{t+1}}{1-\rho}\right)\right], t \geq 2
\end{aligned}
$$

In these equations, note that we have as expected (Vallois and Tapiero [2007]):

$$
\text { (18) } E(x(t))=\frac{\alpha}{1-\rho}\left[t+1-\frac{1-\rho^{t+1}}{1-\rho}\right]
$$

This clearly indicates the nonlinear time effects of persistence in such counting processes. A verification of (17) can also be reached. First note that when there is no persistence, then $\rho=0$ and $E\left(x(t)^{2}\right)=E\left(x(t-1)^{2}\right)+\alpha[1+2 \alpha(t-1)]$. Summing for $1 \leq t \leq n$, we obtain $E\left(x(n)^{2}\right)=n \alpha(1+\alpha(n-1))$. Since $x(n)$ has a binomial distribution $B(n, \alpha)$, we have $E(x(n))=n \alpha, \quad V \operatorname{ar}(x(n))=n \alpha(1-\alpha)$ and thereby: $E\left(x(n)^{2}\right)=n \alpha(1-\alpha)+n^{2} \alpha^{2}=n \alpha(1+(n-1) \alpha)$ as expected. 
Explicit expressions for the persistent counting probabilities can be determined as well using the recursive probability generating functions. In this case, we calculate $\left(p_{k}(t) ; 0 \leq k \leq t\right)$ by recurrence. Initially these are specified by:

(19) $p_{0}(0)=1, p_{0}(1)=1-\alpha, p_{1}(1)=\alpha$

Further, $\left(p_{k}(t+2) ; 0 \leq k \leq t+2\right)$ is defined as a function of $\left(p_{k}(t+1) ; 0 \leq k \leq t+1\right)$ and $\left(p_{k}(t) ; 0 \leq k \leq t\right)$ by using the recursive equation

(20) $\quad p_{k}(t+2)=(1-\alpha) p_{k}(t+1)+(\alpha+\rho) p_{k-1}(t+1)-\rho p_{k-1}(t)$,

for all $0 \leq k \leq t+2$, and by convention, we set $p_{k}(t)=0$ if $k<0$ or $k>t$.

Subsequent calculations will indicate the underlying process probabilities. In particular, we have for the first 3 probabilities:

$$
\begin{aligned}
p_{0}(t) & =(1-\alpha)^{t} \\
\text { (21) } p_{1}(t) & =\alpha(1-\alpha)^{t-2}((1-\alpha-\rho) t+\rho), t \geq 1 \\
p_{2}(t) & =\alpha\left\{\begin{array}{l}
(\alpha+\rho)(1-\alpha)^{t-2} \\
+\frac{1}{2}(1-\alpha)^{t-4}(1-\alpha-\rho)(t-2)\left(\alpha(1-\alpha-\rho) t+\alpha+2 \rho-\alpha^{2}+\alpha \rho\right)
\end{array}\right\}, t \geq 2
\end{aligned}
$$

Of course, when there is no persistence, this is reduced as expected to:

$$
\begin{aligned}
p_{0}(t) & =(1-\alpha)^{t} \\
p_{1}(t) & =\alpha t(1-\alpha)^{t-1}, t \geq 1 \\
p_{2}(t) & =\frac{1}{2} \alpha^{2}(1-\alpha)^{t-2} t(t-1), t \geq 2
\end{aligned}
$$

\section{Application: The Compound Counting Persistent Process}

In many applications, counting is used for summing events that are independent (for example, the Compound Poisson processes). Expectedly, when counting is persistent (and therefore dependent), such a statistical characteristic has to be accounted for. The analytical results regarding the counting persistent process can be used and applied to numerous problems. For example, say that an insurance firm seeks to calculate the sum of claims (independently distributed but dependent on the persistent counting process-and therefore the Poisson distribution cannot be used). Let the sum of claims be $\xi_{t}=\sum_{j=1}^{x_{t}} \tilde{Z}_{j}$ where claims 
$\tilde{Z}_{i}$ are statistically independent with mean $\mu=E\left(\tilde{Z}_{i}\right)$ and known variance $\operatorname{var}\left(\tilde{Z}_{i}\right)$. Then, for independent (Poisson) counting processes, the mean claims up to time $\mathrm{t}$ are:

$$
E\left(\sum_{i=1}^{x_{t}} \tilde{Z}_{i}\right)=E\left(x_{t}\right) E\left(\tilde{Z}_{i}\right)
$$

while claims variance is given by:

$$
\operatorname{var}\left(\sum_{i=1}^{x_{t}} \tilde{Z}_{i}\right)=\left[E\left(\tilde{Z}_{i}\right)\right]^{2} \operatorname{Var}\left(x_{t}\right)+\operatorname{Var}\left(\tilde{Z}_{i}\right) E\left(x_{t}\right) \text {. }
$$

However, when claims are persistence-dependent a more general expression can be found. Explicitly, consider the following random claims:

(23) $S_{t}=\sum_{i=0}^{t} \tilde{Z}_{i}^{0} 1_{\left\{y_{i}=0\right\}}+\sum_{i=0}^{t} \tilde{Z}_{i}^{1} 1_{\left\{y_{i}=1\right\}}$ where $x_{t}=\sum_{i=0}^{t} y_{i}$ where $\tilde{Z}_{i}^{0}$ is a "normal claim" occurring in any regular period (defined by the fact that no specific event has occurred) while $\tilde{Z}_{i}^{1}$ is a "large claim" (of course, if $\tilde{Z}_{i}^{0}=\tilde{Z}_{i}^{1}$ then $S_{t}=\xi_{t}$ as stated above). We assume that $\left\{\tilde{Z}_{i}^{0}, \tilde{Z}_{i}^{1}\right\}$ are random variables independent of each other and independent of the Markov (persistent) claims. In this case, the compound claim mean and variance and the claim probability generating function are given by the following (with proofs provided in the appendix):

$$
\begin{aligned}
E\left(S_{t}\right) & =(t+1) E\left(\tilde{Z}_{1}^{0}\right)+\left(E\left(\tilde{Z}_{1}^{1}\right)-E\left(\tilde{Z}_{1}^{0}\right)\right) E\left(x_{t}\right) \\
E\left(S_{t}^{2}\right) & =(t+1)\left[\operatorname{var}\left(\tilde{Z}_{1}^{0}\right)+(t+1)\left(E\left(\tilde{Z}_{1}^{0}\right)\right)^{2}\right]+E\left(x_{t}^{2}\right)\left[E\left(\tilde{Z}_{1}^{1}\right)-E\left(\tilde{Z}_{1}^{0}\right)\right]^{2} \\
& +E\left(x_{t}\right)\left[\operatorname{var}\left(\tilde{Z}_{1}^{1}\right)-\operatorname{var}\left(\tilde{Z}_{1}^{0}\right)+2(t+1) E\left(\tilde{Z}_{1}^{0}\right)\left(E\left(\tilde{Z}_{1}^{1}\right)-E\left(\tilde{Z}_{1}^{0}\right)\right)\right]
\end{aligned}
$$

While their Laplace Transform is:

$$
\text { (26) } E\left(e^{-\lambda S_{t}}\right)=\left[E\left(e^{-\lambda \tilde{Z}_{i}^{0}}\right)\right]^{t+1} G(z, t), \quad z=\frac{E\left(e^{-\lambda \hat{Z_{1}^{1}}}\right)}{E\left(e^{-\lambda \tilde{Z}_{1}^{0}}\right)}, \lambda \geq 0
$$

where $G(z, t)$ is a probability generating function:

$$
G(z, t)=E\left(z^{x_{t}}\right)=\sum_{i=0}^{t+1} z^{i} P\left(x_{t}=i\right) .
$$


Note that when $\tilde{Z}_{1}^{0}$ is null, then, from equation (26) we find that:

$$
\text { (27) } E\left(S_{t}\right)=E\left(\tilde{Z}_{1}^{1}\right) E\left(x_{t}\right) \text { and } \operatorname{Var}\left(S_{t}\right)=E\left(x_{t}\right) \operatorname{Var}\left(\tilde{Z}_{1}^{1}\right)+\operatorname{Var}\left(x_{t}\right)\left(E\left(\tilde{Z}_{1}^{1}\right)\right)^{2}
$$

These results can then be used to obtain approximate prices for the premium to be charged (based on mean-variance rules) when the counting process is persistent. Note however that the variance of this process has increased over time due to the process persistence. Further, based on these moments, the Value at Risk (VaR) risk exposure can be determined which would use these two moments as a first approximation (although higher order moments can be calculated as well using (26), the generating function of the persistent Compound Process).

\section{Some Numerical Results}

A numerical analysis of our equations will reveal some of the characteristics of a persistent process. As expected, the mean evolution of the persistent process has an almost linear growth as indicated in our equation. In the long run, the variance turns out to be also almost linear, as it is the case for random walks. However, persistence $(\rho>0)$ has the effect of increasing the variance as shown in Figure-1 below. In the short term however, the variance evolution is nonlinear as our equations have indicated.

Interestingly, the rate of change in variance is not constant and growing over time which indicates a "persistent volatility". Of particular interest is the evolution of the third moment of the persistent claim distribution. Initially, it was increasing (over 4 periods) and subsequently declining (although remaining positive for $\rho$ positive). When $\rho$ is negative we note that for the first few periods the evolution of the mean and the variance are indeed nonlinear. This is particularly the case for the variance as shown in Figure 2 below. In this Figure, the variance initially declines, then increases and again decreases. Finally, it converged to a linear growth. This behavior is indicative of the short term effects of memory on the stochastic process as indicated earlier. In Figure 3 , we note the divergence in the growth of volatility when the persistence parameter is negative. Finally, the third moment is positive which demonstrate that the distribution is skewed reflecting the process memory- 
persistence. This latter observation is particularly important for it may be used to explain partly the skew of certain time series, presuming that this skew is due to the short term memory effects prevalent in such series (for example, in financial time series).

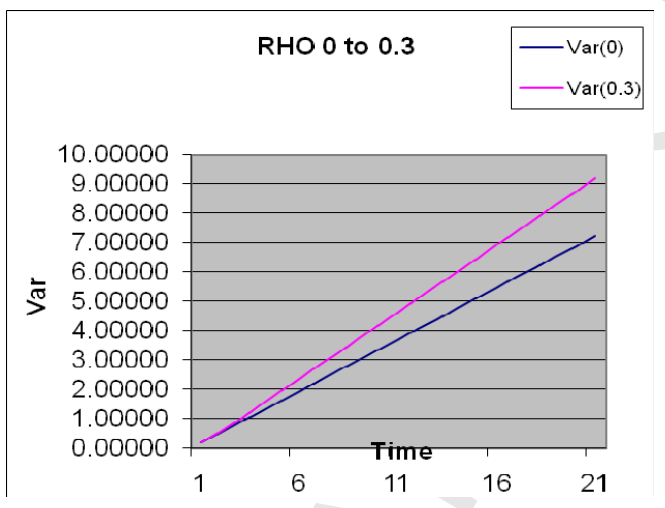

Figure 1: Persistence and Variance of the Counting process

Figure 2 : $\mathrm{Rho}=-0.3$

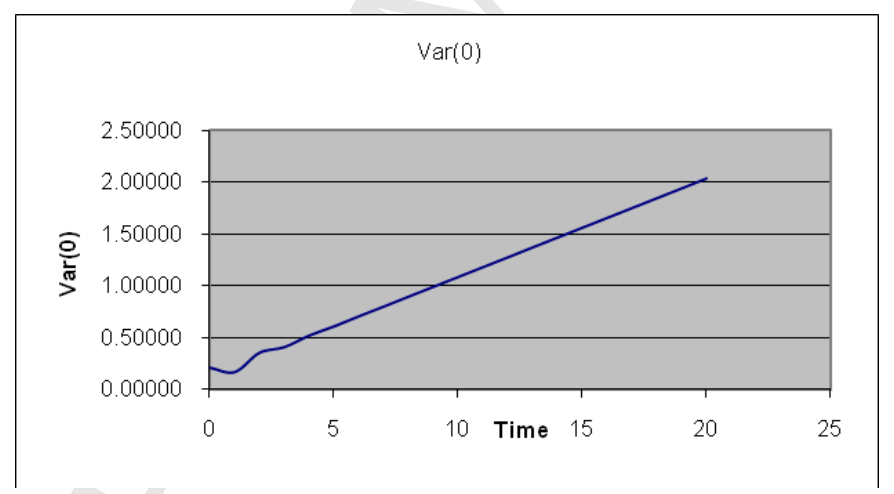

Figure 3: Rho=-0.3 


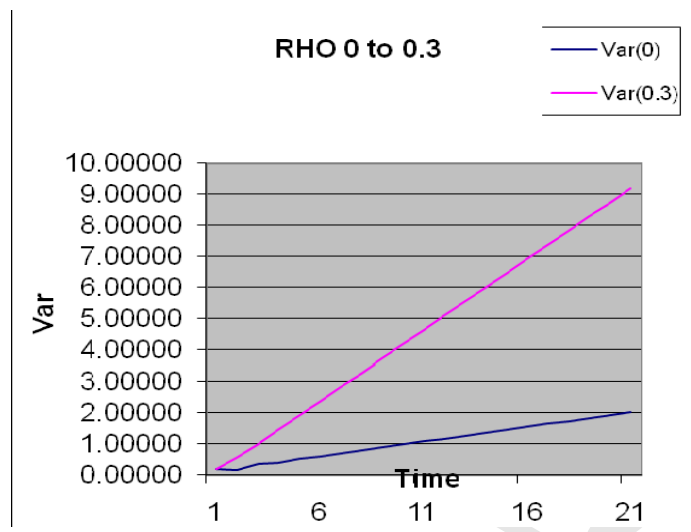

Similarly, numerical analysis of some of the first persistent probabilities reveal a certain cyclicality, a function of the peristent indexr. As shown in Figures 4 and 5, the evolution of the claim probability is complex when there is persistence (memory) compared to that without persistence, oscillating initially and subsequently converging.

Figure 4: The Probability of a Claim, $\rho=-0.3$

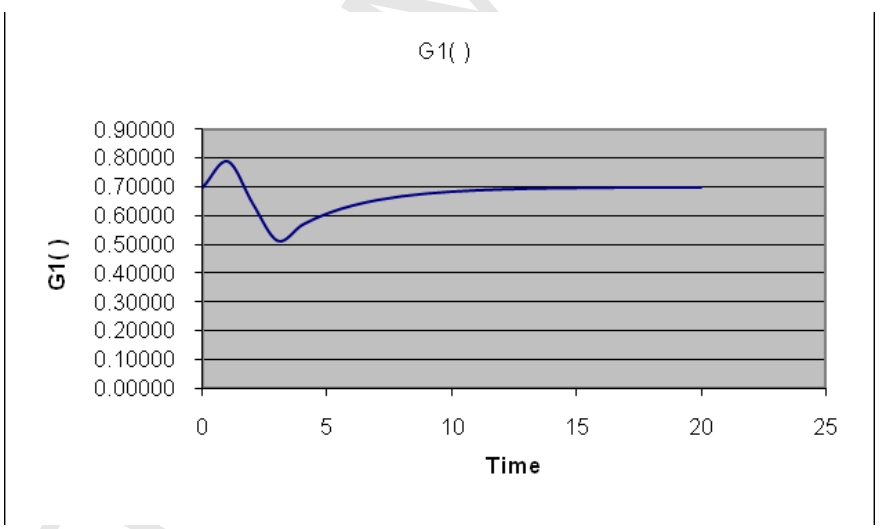


Figure 5: The Probability of a Claim, $\rho=0.3$

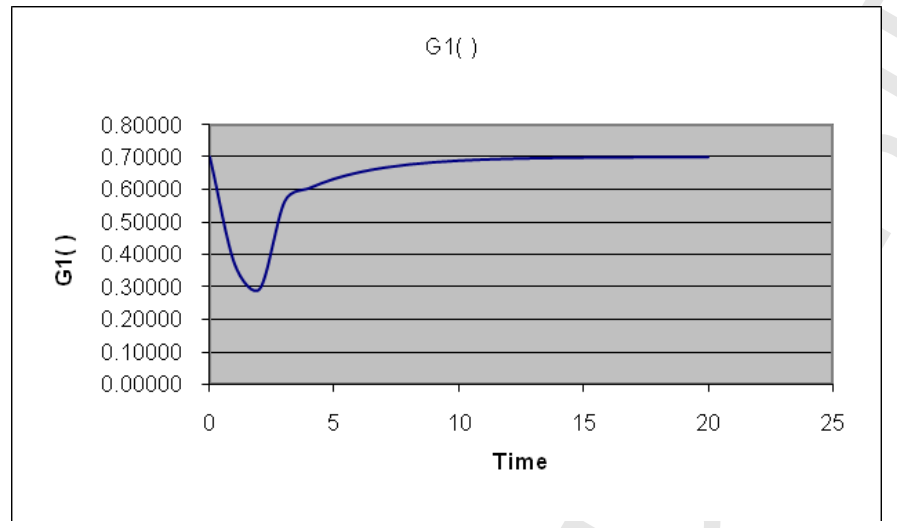

\section{Conclusion}

The persistent counting process in insurance is both of theoretical and practical importance. Such a process, appropriately structured and based on the relevant data that insurance companies possess may be used as an underlying process with respect to which premiums, risk exposure and risk management procedures may be determined when persistence is inherent to the insurance environment. While applications to insurance were emphasized, the results obtain here are equally applicable to numerous problems where counting processes are persistent. Further research on the implications of this approach to multiple periods memories as well as the effects of memory on skewness and process cyclicality are needed however. The numerical results and the moments calculations in this paper have indicated however that persistence matters and leads to numerous stochastic phenomena that are observed in practice and hardly explained.

\section{References}

Balinth Toth, 1986, Persistent random walks in random environments, Probability Theory and Related Fields, 71, 615-625

Claes I. and C. Van den Broeck, 1987, Random Walk with persistence, Journal of Statistical Physics, vol. 49, No.1-2, October, pp. 383-392.

Denuit Michel, Dhaene, Jan, Goovaerts, Marc and Kaas, Rob, Actuarial Theory for Dependent Risks: Measures, Orders and Models, ISBN: 978-0-470-01644-2, Adobe E-Book, 458 pages, June 2006 
Patlak, Clifford, 1953, Random walk with persistence and external bias, Bulletin of Mathematical Biology, vol. 15, no. 3, September, pp. 311-338

Pottier, Noelle, 1996, Analytic study of the effect of persistence on a one-dimensional biased random walk, Physica A 230 (1996) 563-576

Telesca L. and M. Lovallo, 2006, Are global terrorist attacks time correlated, Physica A, 362, 480-484

Vallois P. and C. S. Tapiero, 2007, Memory-Based Persistence in A Counting Random Walk Process, Physica A (Statistical Mechanics)

Weiss, G.H., 1994, Aspects and Applications of the Random Walk, NorthHolland, Amsterdam.

Weiss G.H. 2002, Some applications of persistent random walks and the telegrapher's equation, Physica A, 311, 381-410

Weiss, G.H., and R.J. Rubin, 1983, Random walks: theory and selected applications, Adv Chemical Physics, 52, 363-505 


\section{Appendix 1: Proof of Proposition 1}

Let

$$
F_{0}(\lambda, t)=E\left\{\lambda^{x_{t}} 1_{\left\{y_{t}=0\right\}}\right\} \text { and } F_{1}(\lambda, t)=E\left\{\lambda^{x_{t}} 1_{\left\{y_{t}=1\right\}}\right\}
$$

And therefore,

$$
F_{0}(\lambda, t)=E\left\{\lambda^{x_{t-1}+y_{t}} 1_{\left\{y_{t}=0\right\}}\right\}=E\left\{\lambda^{x_{t-1}} 1_{\left\{y_{t-1}=0\right\}}\right\}(1-\alpha)+E\left\{\lambda^{x_{t-1}} 1_{\left\{y_{t-1}=1\right\}}\right\} \beta .
$$

Similarly,

$$
F_{1}(\lambda, t)=E\left\{\lambda^{x_{t}} 1_{\left\{y_{t}=1\right\}}\right\}=E\left\{\lambda^{x_{t-1}+1} 1_{\left\{y_{t}=1\right\}}\right\}=\lambda E\left\{\lambda^{x_{t-1}} 1_{\left\{y_{t-1}=0\right\}}\right\} \alpha+\lambda E\left\{\lambda^{x_{t-1}} 1_{\left\{y_{t-1}=1\right\}}\right\}(1-\beta) .
$$

As a result, we can write:

$$
\left(\begin{array}{l}
F_{0}(\lambda, t) \\
F_{1}(\lambda, t)
\end{array}\right)=\left(\begin{array}{cc}
1-\alpha & \beta \\
\lambda \alpha & \lambda(1-\beta)
\end{array}\right)\left(\begin{array}{l}
F_{0}(\lambda, t-1) \\
F_{1}(\lambda, t-1)
\end{array}\right)
$$

Or in a matrix notation:

$$
\mathbf{F}(\lambda, \mathbf{t})=\mathbf{A}^{\mathbf{t}} \mathbf{F}(\lambda, \mathbf{0}) ; \quad \mathbf{A}=\left(\begin{array}{cc}
1-\alpha & \beta \\
\lambda \alpha & \lambda(1-\beta)
\end{array}\right) ; \quad \mathbf{F}(\lambda, \mathbf{t})=\left(\begin{array}{l}
F_{0}(\lambda, t) \\
F_{1}(\lambda, t)
\end{array}\right)
$$

Note that the matrix eigenvalues $\mu$ are a solution to:

$$
(1-\alpha-\mu)(\lambda(1-\beta)-\mu)-\lambda \alpha \beta=0
$$

For convenience, define $\rho=1-\alpha-\beta$, then

$$
(1-\alpha-\mu)(\lambda(\rho+\alpha)-\mu)-\lambda \alpha(1-\alpha-\rho)=0
$$

and the eigenvalues are a solution of the quadratic equation:

$$
\mu^{2}-\mu(\lambda(\rho+\alpha)+1-\alpha)+\lambda \rho=0
$$

Setting $a=\lambda \alpha+1-\alpha$ we have instead $\mu^{2}-\mu(a+\lambda \rho)+\lambda \rho=0$ as a result:

$$
\mu_{i}=\frac{a+\lambda \rho}{2} \pm \frac{1}{2} \sqrt{(a+\lambda \rho)^{2}-4 \lambda \rho}, i=0,1
$$

Or

$$
\begin{aligned}
& \mu_{0}=\frac{a+\lambda \rho}{2}+\frac{1}{2} \sqrt{(a+\lambda \rho)^{2}-4 \lambda \rho}, \\
& \mu_{1}=\frac{a+\lambda \rho}{2}-\frac{1}{2} \sqrt{(a+\lambda \rho)^{2}-4 \lambda \rho}
\end{aligned}
$$

And 


$$
\mu_{0}+\mu_{1}=a+\lambda \rho, \mu_{0}-\mu_{1}=\sqrt{\delta}, \delta=(a+\lambda \rho)^{2}-4 \lambda \rho
$$

Initial conditions are found by:

$$
\begin{aligned}
& F_{0}(\lambda, 0)=E\left(\lambda^{x_{0}} 1_{\left\{x_{0}=0\right\}}\right)=P\left(x_{0}=0\right) . \\
& F_{1}(\lambda, 0)=E\left(\lambda^{x_{0}} 1_{\left\{x_{0}=1\right\}}\right)=\lambda P\left(x_{0}=1\right) .
\end{aligned}
$$

While

$$
F_{0}(\lambda, 1)=E\left(\lambda^{x_{1}} 1_{\left\{x_{1}=0\right\}}\right)=(1-\alpha) F_{0}(\lambda, 0)+\beta F_{1}(\lambda, 0) .
$$

And therefore

$$
F_{0}(\lambda, 1)=(1-\alpha) P\left(x_{0}=0\right)+\lambda \beta P\left(x_{0}=1\right) .
$$

Which we rewrite conveniently by replacing $\beta$ by $1-\alpha-\rho$, or:

$$
F_{0}(\lambda, 1)=(1-\alpha) P\left(x_{0}=0\right)+\lambda(1-\alpha-\rho) P\left(x_{0}=1\right) .
$$

Similarly,

$$
F_{1}(\lambda, 1)=\lambda \alpha F_{0}(\lambda, 0)+\lambda(1-\beta) F_{1}(\lambda, 0) .
$$

And therefore

$$
F_{1}(\lambda, 1)=\lambda \alpha P\left(x_{0}=0\right)+\lambda^{2}(\rho+\alpha) P\left(x_{0}=1\right) .
$$

Since from the solution of our matrix equations we have:

$$
F_{0}(\lambda, t)=u_{0} \mu_{0}^{t}+u_{1} \mu_{1}^{t} \text { and } F_{1}(\lambda, t)=v_{0} \mu_{0}^{t}+v_{1} \mu_{1}^{t} .
$$

Where $\left(u_{0}, u_{1}, v_{0}, v_{1}\right)$ are parameters calculated by the initial conditions we have defined previously. As a result, we have:

$$
\begin{aligned}
& F_{0}(\lambda, 0)=u_{0}+u_{1} \\
& F_{0}(\lambda, 1)=u_{0} \mu_{0}+u_{1} \mu_{1}
\end{aligned} \text { and } \begin{aligned}
& F_{1}(\lambda, 0)=v_{0}+v_{1} \\
& F_{1}(\lambda, 1)=v_{0} \mu_{0}+v_{1} \mu_{1}
\end{aligned}
$$

These lead to the following solutions:

$$
\begin{aligned}
& u_{0}=\frac{F_{0}(\lambda, 1)-F_{0}(\lambda, 0) \mu_{1}}{\mu_{0}-\mu_{1}}=\frac{F_{0}(\lambda, 1)-F_{0}(\lambda, 0) \mu_{1}}{\sqrt{\delta}} . \\
& u_{1}=\frac{F_{0}(\lambda, 0) \mu_{0}-F_{0}(\lambda, 1)}{\mu_{0}-\mu_{1}}=\frac{F_{0}(\lambda, 0) \mu_{0}-F_{0}(\lambda, 1)}{\sqrt{\delta}} .
\end{aligned}
$$

By the same token:

$$
v_{0}=\frac{F_{1}(\lambda, 1)-F_{1}(\lambda, 0) \mu_{1}}{\sqrt{\delta}}, \quad v_{1}=\frac{F_{1}(\lambda, 0) \mu_{0}-F_{1}(\lambda, 1)}{\sqrt{\delta}}
$$




$$
\begin{aligned}
& F_{0}(\lambda, t)=\frac{F_{0}(\lambda, 1)-F_{0}(\lambda, 0) \mu_{1}}{\sqrt{\delta}} \mu_{0}^{t}+\frac{F_{0}(\lambda, 0) \mu_{0}-F_{0}(\lambda, 1)}{\sqrt{\delta}} \mu_{1}^{t} \\
& F_{1}(\lambda, t)=\frac{F_{1}(\lambda, 1)-F_{1}(\lambda, 0) \mu_{1}}{\sqrt{\delta}} \mu_{0}^{t}+\frac{F_{1}(\lambda, 0) \mu_{0}-F_{1}(\lambda, 1)}{\sqrt{\delta}} \mu_{1}^{t}
\end{aligned}
$$

Since, $G(\lambda, t)=E\left[\lambda^{x_{t}}\right]=F_{0}(\lambda, t)+F_{1}(\lambda, t)$, we obtain the following probability generating function:

$$
\begin{aligned}
G(\lambda, t) & =\left(\frac{\left(F_{0}(\lambda, 1)+F_{1}(\lambda, 1)\right)-\left(F_{0}(\lambda, 0)+F_{1}(\lambda, 0)\right) \mu_{1}}{\sqrt{\delta}}\right) \mu_{0}^{t} \\
& +\left(\frac{\left(F_{0}(\lambda, 0)+F_{1}(\lambda, 0)\right) \mu_{0}-\left(F_{0}(\lambda, 1)+F_{1}(\lambda, 1)\right)}{\sqrt{\delta}}\right) \mu_{1}^{t}
\end{aligned}
$$

Or expressed in terms of the known initial conditions

$$
G(\lambda, t)=\left(\frac{G(\lambda, 1)-G(\lambda, 0) \mu_{1}}{\sqrt{\delta}}\right) \mu_{0}^{t}+\left(\frac{G(\lambda, 0) \mu_{0}-G(\lambda, 1)}{\sqrt{\delta}}\right) \mu_{1}^{t} .
$$

Which can be rewritten by:

$$
G(\lambda, t)=\frac{G(\lambda, 1)}{\sqrt{\delta}}\left(\mu_{0}^{t}-\mu_{1}^{t}\right)-\frac{G(\lambda, 0) \mu_{0} \mu_{1}}{\sqrt{\delta}}\left(\mu_{0}^{t-1}-\mu_{1}^{t-1}\right) .
$$

Note that $\mu_{0} \mu_{1}=\lambda \rho$ and thereby, in a recursive form, we have:

$$
G(\lambda, t)=\frac{G(\lambda, 1)}{\sqrt{\delta}} \Psi_{t}-\frac{\lambda \rho G(\lambda, 0)}{\sqrt{\delta}} \Psi_{t-1}, \Psi_{t}=\mu_{0}^{t}-\mu_{1}^{t}
$$

As stated in the proposition. In addition:

$$
G(\lambda, 0)=F_{0}(\lambda, 0)+F_{1}(\lambda, 0)=P\left(x_{0}=0\right)+\lambda P\left(x_{0}=1\right)
$$

and

$$
G(\lambda, 1)=a P\left(x_{0}=0\right)+\lambda P\left(x_{0}=1\right)+\lambda(\lambda-1)(\alpha+\rho) P\left(x_{0}=1\right)
$$

As stated in Proposition 1. 


\section{Appendix 2}

Since the random variables $\left\{\tilde{Z}_{i}^{0}, \tilde{Z}_{i}^{1}\right\}$ are independent of $y_{t}$, we have:

$$
E\left(S_{t}\right)=\sum_{i=0}^{t} E\left(\tilde{Z}_{i}^{0}\right) P\left(y_{i}=0\right)+\sum_{i=0}^{t} E\left(\tilde{Z}_{i}^{1}\right) P\left(y_{i}=1\right)
$$

However, $E\left(\tilde{Z}_{i}^{0}\right)=E\left(\tilde{Z}_{1}^{0}\right)$ and $E\left(\tilde{Z}_{i}^{1}\right)=E\left(\tilde{Z}_{1}^{1}\right), \forall i \geq 1$, consequently,

$$
E\left(S_{t}\right)=E\left(\tilde{Z}_{1}^{0}\right) \sum_{i=0}^{t}\left(1-P\left(y_{i}=1\right)\right)+E\left(\tilde{Z}_{1}^{1}\right) \sum_{i=0}^{t} P\left(y_{i}=1\right)
$$

And

$$
E\left(S_{t}\right)=(t+1) E\left(\tilde{Z}_{1}^{0}\right)+\left(E\left(\tilde{Z}_{1}^{1}\right)-E\left(\tilde{Z}_{1}^{0}\right)\right) \sum_{i=0}^{t} P\left(y_{i}=1\right)
$$

But $E\left(x_{t}\right)=\sum_{i=0}^{t} P\left(y_{i}=1\right)$ which proves (2).

In order to calculate the variance of the Compound persistent claim process we first state the following Lemma:

\section{Lemma 1:}

Conditionally on $x_{t}=k$, the Compound Claim $S_{t}$ is distributed as:

$$
\sum_{i=1}^{k} \tilde{Z}_{i}^{1}+\sum_{i=1}^{t+1-k} \tilde{Z}_{i}^{0}
$$

\section{Proof:}

Let $i_{0}, i_{1}, \ldots, i_{t}$ be a series assuming values 0 and 1 and let $\mathrm{k}$ be the number the index $\mathrm{j}$ assumes a value $1, i_{j}=1$. Conditionally on $y_{0}=i_{0}, y_{1}=i_{1}, \ldots, y_{t}=i_{t}$. we have by the definition of $x_{t}=k$, that:

$$
S_{t}=\sum_{j, i_{j}=1}^{k} \tilde{Z}_{i_{j}}^{1}+\sum_{j, i_{j}=0}^{t+1-k} \tilde{Z}_{i_{j}}^{0}
$$

The first sum consists of $k$ terms while the second consists of $t+1-k$ terms. As a result, their sum is distributed as defined in the Lemma. 
These results lead to the following Lemma, expressing the expected second moment:

Lemma 2:

$$
\begin{aligned}
E\left(S_{t}^{2}\right) & =(t+1)\left[\operatorname{var}\left(\tilde{Z}_{1}^{0}\right)+(t+1)\left(E\left(\tilde{Z}_{1}^{0}\right)\right)^{2}\right] \\
& +E\left(x_{t}\right)\left[\operatorname{var}\left(\tilde{Z}_{1}^{1}\right)-\operatorname{var}\left(\tilde{Z}_{1}^{0}\right)+2(t+1) E\left(\tilde{Z}_{1}^{0}\right)\left(E\left(\tilde{Z}_{1}^{1}\right)-E\left(\tilde{Z}_{1}^{0}\right)\right)\right] \\
& +E\left(x_{t}^{2}\right)\left[E\left(\tilde{Z}_{1}^{1}\right)-E\left(\tilde{Z}_{1}^{0}\right)\right]^{2}
\end{aligned}
$$

Proof:

$$
\begin{aligned}
& E\left(S_{t}^{2} \mid x_{t}=k\right)=E\left(\sum_{i=1}^{k} \tilde{Z}_{1}^{1}+\sum_{i=1}^{t+1-k} \tilde{Z}_{1}^{0}\right)^{2} \\
& =\operatorname{Var}\left(\sum_{i=1}^{k} \tilde{Z}_{1}^{1}+\sum_{i=1}^{t+1-k} \tilde{Z}_{1}^{0}\right)+\left[E\left(\sum_{i=1}^{k} \tilde{Z}_{1}^{1}+\sum_{i=1}^{t+1-k} \tilde{Z}_{1}^{0}\right)\right]^{2} \\
& =k \operatorname{Var}\left(\tilde{Z}_{1}^{1}\right)+(t+1-k) \operatorname{Var}\left(\tilde{Z}_{1}^{0}\right)+\left(k E\left(\tilde{Z}_{1}^{1}\right)+(t+1-k) E\left(\tilde{Z}_{1}^{0}\right)\right)^{2} \\
& =(t+1) \operatorname{Var}\left(\tilde{Z}_{1}^{0}\right)+k\left(\operatorname{Var}\left(\tilde{Z}_{1}^{1}\right)-\operatorname{Var}\left(\tilde{Z}_{1}^{0}\right)\right)+\left\{(t+1) E\left(\tilde{Z}_{1}^{0}\right)+k\left(E\left(\tilde{Z}_{1}^{1}\right)-E\left(\tilde{Z}_{1}^{0}\right)\right)\right\}^{2}
\end{aligned}
$$

Thus

$$
\begin{aligned}
E\left(S_{t}^{2} \mid X_{t}=\right. & k)=(t+1)\left(\operatorname{Var}\left(\tilde{Z}_{1}^{0}\right)+(t+1)\left[E\left(\tilde{Z}_{1}^{0}\right)\right]^{2}\right) \\
& +k\left(\operatorname{Var}\left(\tilde{Z}_{1}^{1}\right)-\operatorname{Var}\left(\tilde{Z}_{1}^{0}\right)+2(t+1) E\left(\tilde{Z}_{1}^{0}\right)\left(E\left(\tilde{Z}_{1}^{1}\right)-E\left(\tilde{Z}_{1}^{0}\right)\right)\right) \\
& +k^{2}\left[E\left(\tilde{Z}_{1}^{1}\right)-E\left(\tilde{Z}_{1}^{0}\right)\right]^{2}
\end{aligned}
$$

Since

$$
E\left(S_{t}^{2}\right)=\sum_{k \geq 0} E\left(S_{t}^{2} \mid x_{t}=k\right) P\left(x_{t}=k\right),
$$

We obtain:

$$
\begin{aligned}
E\left(S_{t}^{2}\right)=( & t+1)\left(\operatorname{Var}\left(\tilde{Z}_{1}^{0}\right)+(t+1)\left[E\left(\tilde{Z}_{1}^{0}\right)\right]^{2}\right) \\
& +E\left(x_{t}\right)\left(\operatorname{Var}\left(\tilde{Z}_{1}^{1}\right)-\operatorname{Var}\left(\tilde{Z}_{1}^{0}\right)+2(t+1) E\left(\tilde{Z}_{1}^{0}\right)\left(E\left(\tilde{Z}_{1}^{1}\right)-E\left(\tilde{Z}_{1}^{0}\right)\right)\right) \\
& +E\left(x_{t}^{2}\right)\left[E\left(\tilde{Z}_{1}^{1}\right)-E\left(\tilde{Z}_{1}^{0}\right)\right]^{2}
\end{aligned}
$$

As stated in the Lemma. 
Finally, using the same procedure above, and for positive claims (random variables $\left.\left(\tilde{Z}_{i}^{0} \geq 0, \tilde{Z}_{i}^{1} \geq 0, S_{t} \geq 0\right)\right)$ the Laplace Transform of the persistent Compound Poisson process can be calculated as well. In this case, we have the following:

\section{Lemma 3}

Let $\lambda \geq 0$, then: $E\left(e^{-\lambda S_{t}}\right)=\left[E\left(e^{-\lambda \tilde{z}_{i}^{0}}\right)\right]^{t+1} G(z, t), z=\frac{E\left(e^{-\lambda \tilde{z}_{1}^{1}}\right)}{E\left(e^{-\lambda \tilde{z}_{1}^{0}}\right)}$ With

$G(z, t)=E\left(z^{x_{t}}\right)=\sum_{i=0}^{t+1} z^{i} P\left(x_{t}=i\right)$

Proof:

The proof is based on Lemma 1 and follows a procedure similar to Lemma 2. Namely,

$$
\begin{aligned}
& E\left(e^{-\lambda S_{t}} \mid x_{t}=k\right)=E\left(e^{-\lambda\left(\tilde{Z}_{1}^{1}+\ldots+\ldots \tilde{Z}_{k}^{1}+\tilde{Z}_{1}^{0}, \ldots, \tilde{Z}_{t+1-k}^{0}\right)}\right)=\left[E\left(e^{-\lambda \tilde{Z}_{1}^{1}}\right)\right]^{k}\left[E\left(e^{-\lambda \tilde{Z}_{1}^{0}}\right)\right]^{t+1-k} \\
& =\left[E\left(e^{-\lambda \tilde{Z}_{1}^{0}}\right)\right]^{t+1}\left[\frac{E\left(e^{-\lambda \tilde{Z}_{1}}\right)}{E\left(e^{-\lambda \tilde{Z}_{1}^{0}}\right)}\right]^{k}
\end{aligned}
$$

Further, it is evident that:

$$
E\left(e^{-\lambda S_{t}}\right)=\sum_{k \geq 0} E\left(e^{-\lambda S_{t}} \mid x_{t}=k\right) P\left(x_{t}=k\right)
$$

which provides the desired result stated in the Lemma above. 